\title{
FROM THE EDITORS' DESK
}

\section{Patient-Reported Outcomes as a Measure of Healthcare Quality}

\author{
Dominick L. Frosch, $P h D^{1,2}$ \\ 'Patient Care Program, Gordon and Betty Moore Foundation, Palo Alto, CA, USA; ${ }^{2}$ Department of Medicine, University of California, Los Angeles, \\ Los Angeles, CA, USA.
}

J Gen Intern Med 30(10): 1383-4

DOI: $10.1007 / \mathrm{s} 11606-015-3476-2$

(c) Society of General Internal Medicine 2015

$\mathrm{T}$ he technical minutiae and nuances of assessing and measuring the quality of healthcare can, at times, seem far removed from broad conceptual and philosophical discussions about what patients want from their healthcare providers. But as our healthcare system undergoes a fundamental shift, reorienting payment for healthcare services from volume to value, it is worth stepping back to consider how we can link the quality of care to the meaning of life.

Consider this perspective. In a 1994 article, Robert Kaplan, one of the pioneers of patient-reported outcome measurement, quotes a Ziggy cartoon by Tom Wilson. In the cartoon, a wise old man is asked about the meaning of life. He replies, "Ah, yes... the meaning of life. ...Life, my boy, is doin' stuff! As opposed to death, which is not doin' stuff." At first blush, this statement seems almost so banal as to be meaningless, and as any clinician knows, there's plenty of room for variation between life and death. But then again, doing stuff is the fundamental building block by which each of us creates meaning in our lives. From the patient's perspective, medical care is fundamentally about restoring or maintaining our ability to do stuff, to be free of functional impairments or symptoms that interfere with our ability to pursue our life goals. ${ }^{1}$

This is where patient-reported outcomes enter the picture. These measures capture patients' subjective experience of illness, impairment and disability, and are increasingly used in research to evaluate therapies. The initial objective in developing these measures was to go beyond simple outcome measures like mortality, to be able to capture the difference between a tennis player (someone who in Ziggy's terms is "doin' stuff") and someone in a coma, who would not be captured by measures of mortality, but without a doubt is suffering a very significant impairment in their ability to "do stuff." Over the last 40 years, the science of patient-reported outcome measures, largely driven by their use in research and clinical trials, has advanced to a mature stage. Mountains of evidence document the validity of these subjective measures.

Published online July 30, 2015
Even a simple, single question that asks an individual to rate their health from poor to excellent has strong predictive validity for healthcare utilization and for mortality. ${ }^{2}$ At the same time, patient-reported outcome measures (PROMs) have also clearly shown that improving clinical endpoints (e.g., biomarkers of illness or disease activity) does not always translate into subjective improvement for patients. ${ }^{1,3}$ In fact, there are many examples of treatments that significantly alter surrogate biological markers, but have no effect on outcomes of interest to patients: mortality and quality of life.

As the science and interest in patient-reported outcome measures have grown, measures have proliferated, raising some challenges. How does one compare results from a clinical trial that used one particular measure to those using a different patient-reported outcome measure? Three papers in this issue of JGIM significantly advance the solution to this problem by demonstrating how various PROMs can be translated into a common score using the PROMIS [Patient Reported Outcomes Measurement Information System] measures developed with funding from the National Institutes of Health. ${ }^{2,45}$ From a research perspective, these methods for translating scores from different measures into a common metric offer great potential for comparing the impact of treatment among different therapies. Beyond using PROMs for research or to inform healthcare resource allocation decisions, one could also envision a future in which clinicians and patients considering various clinical options could directly compare their impact using a common metric before making a therapeutic choice.

An important area for application of patient-reported outcomes is in assessing quality of care. The National Quality Forum (NQF) has endorsed numerous outcome measures to track the performance of the healthcare delivery system, but only a handful derive from patient-reported outcome measures. NQF does not endorse PROMs per se. Instead, evidence is first needed to quantify the kind of changes we should expect to see at the aggregate level within a healthcare delivery organization for a given condition. In other words, if a patient is newly diagnosed with diabetes and is appropriately treated, what level of improvement on a patient-reported outcome measure should we expect to see over what period of time? Leveraging electronic data systems to routinely collect large quantities of patient-reported outcome data would provide a wealth of information that could be used to better judge the quality of care from the patient's perspective and would enable 
the development of new, more patient-relevant performance metrics. Did the prescribed therapy really improve outcomes, or did it just change a biomarker that doesn't translate into an actual subjective improvement for the patient? Was the tradeoff that came with the new drug therapy in the form of side effects really worth it for the patient? Perhaps one reason why Joe and Jane Patient have shown so little interest in using publicly reported quality data is that they aren't reported in metrics that make much sense to patients. Patients seek healthcare because they want to improve their health outcomes. It is often hard for them to see the connection between quality metrics and achieving that goal.

Routine measurement of patient-reported outcomes would also enable a shift to providing goal-oriented care instead of traditional problem-oriented care. The problem focus of healthcare has driven clinical medicine for much of the past century. In many respects it has served us well, but as chronic incurable conditions have proliferated, challenges have also surfaced. ${ }^{6}$ Guidelines, which often serve as the basis for performance metrics, tend to view diseases in isolation. For patients who have multiple chronic conditions, this can become especially problematic, as treatment for one condition can interfere with other conditions, thus failing to achieve the patient's goal of being able to do stuff without functional or symptomatic impairment. ${ }^{3}$ While the challenges of problemoriented care may be most acute among those with multiple chronic conditions, they are no less relevant for patients with individual conditions. For example, an arthroscopic knee surgery may be able to correct the anatomical problem thought to be causing knee pain, but it might not actually reduce the patient's pain.

Patient-reported outcome measures are a key tool for ensuring that the care patients receive actually meets their goals for improving their health. Defining our quality measures in a truly patient-centered way is no longer a fanciful vision. We have the tools, but we must put them to work to leverage their full potential and to align healthcare with how patients define quality, to help answer the question: will this care actually help me feel and function better? At the end of the day, patients want to be able to "do stuff." In order to do stuff, they must first remain alive. Then healthcare must help patients optimize function and reduce symptoms. The articles in this issue are an important step in establishing a common set of metrics that can help track progress toward this goal.

Acknowledgments: Gratitude is expressed to Robert Kaplan, Harvey Fineberg and Lyn Paget for feedback on an earlier draft of this editorial.

Corresponding Author: Dominick L. Frosch, PhD; Patient Care Program, Gordon and Betty Moore Foundation, 1661 Page Mill Road, Palo Alto, CA 94304, USA (e-mail: dominick.frosch@moore.org).

\section{REFERENCES}

1. Kaplan RM. The Ziggy theorem: toward an outcomes-focused health psychology. Health Psychol. 1994;13(6):451-60.

2. Hays RD, Spritzer KL, Thompson WW, Cella D. U.S. General Population Estimate for "Excellent" to "Poor" Self-Rated Health Item. J Gen Intern Med. doi:10.1007/s11606-015-3290-x.

3. Reuben DB, Tinetti ME. Goal-oriented patient care-an alternative health outcomes paradigm. N Engl J Med. 2012;366(9):777-9.

4. Schalet BD, Revicki DA, Cook KF, Krishnan E, Fries JF, Cella D. Establishing a common metric for physical function: Linking the HAQ-DI and SF-36 PF subscale to PROMIS $®$ Physical Function. J Gen Intern Med. doi: 10.1007/s11606-015-3360-0.

5. Schalet BD, Rothrock NE, Hays RD, Kazis LE, Cook KF, Rutsohn JP, Cella D. Linking Physical and Mental Health Summary Scores from the Veterans RAND 12-item Health Survey (VR-12) to the PROMIS $®$ Global Health Scale. J Gen Intern Med. doi:10.1007/s11606-015-3453-9.

6. Mold JW, Blake GH, Becker LA. Goal-oriented medical care. Fam Med. 1991;23(1):46-51. 